\title{
Simulation of wet oxidation of silicon based on the interfacial silicon emission model and comparison with dry oxidation
}

\author{
Masashi Uematsu, ${ }^{\text {a) }}$ Hiroyuki Kageshima, and Kenji Shiraishi \\ NTT Basic Research Laboratories, 3-1 Morinosato Wakamiya, Atsugi, Kanagawa 243-0198, Japan
}

(Received 7 August 2000; accepted for publication 31 October 2000)

\begin{abstract}
Silicon oxidation in wet ambients is simulated based on the interfacial silicon emission model and is compared with dry oxidation in terms of the silicon-atom emission. The silicon emission model enables the simulation of wet oxidation to be done using the oxidant self-diffusivity in the oxide with a single activation energy. The amount of silicon emission from the interface during wet oxidation is smaller than that during dry oxidation. The small emission rate for wet oxidation is responsible for the insignificant initial oxidation enhancement and the linear pressure dependence of the oxidation rate observed in wet oxidation. Using a unified set of parameters, the whole range of oxide thickness is fitted for both (100) and (111) substrates in a wide range of oxidation temperatures $\left(800^{\circ} \mathrm{C}-1200^{\circ} \mathrm{C}\right)$ and pressures $(1-20 \mathrm{~atm})$. (C) 2001 American Institute of Physics.
\end{abstract} [DOI: $10.1063 / 1.1335828]$

\section{INTRODUCTION}

Wet oxidation and dry oxidation have been widely used as silicon oxidation processes. ${ }^{1-6}$ Wet and dry oxidation differ from one another in several points (1) wet oxidation is faster than dry oxidation, ${ }^{3}(2)$ there is less significant initial oxidation enhancement for wet oxidation, ${ }^{3,6}$ and (3) the oxidation rate has a linear pressure dependence for wet oxidation $^{5}$ but a sublinear dependence for dry oxidation. ${ }^{6}$ The first point has been explained by the difference in the properties of oxidants based on the Deal-Grove (D-G) theory; the solubility of water (the oxidant for wet oxidation) in the oxide is about three orders of magnitude larger than that of oxygen (that for dry oxidation). ${ }^{3}$ However, there seems to have been no studies that explain points (2) and (3) in a unified manner. In addition, the D-G theory requires a double activation energy for the parabolic rate constant, which corresponds to the oxidant self-diffusivity in the oxide, for both dry and wet oxidation. ${ }^{5,6}$

The key to solving these problems may be to take interfacial $\mathrm{Si}$-atom emission into account. It is well known that $\mathrm{Si}$ atoms are emitted from the silicon/oxide interface during oxidation. $^{7-12}$ The Si-atom emission should occur during not only dry oxidation but also wet oxidation because the formation of oxidation-induced stacking faults (OSF) and oxidation-enhanced diffusion (OED) are observed for both. ${ }^{13-15}$ We have proposed a basic model in which the $\mathrm{Si}$ atoms emitted to the oxide govern the oxidation rate at the interface due to their high concentration. ${ }^{11,12,16}$ Based on this model, we have explained the initial oxidation enhancement ${ }^{11,12}$ and the sublinear dependence ${ }^{16}$ for dry oxidation using a single activation energy for the oxidant selfdiffusivity. In this article, we apply our model to the simulation of wet oxidation and show that it enables us to do the simulation using the oxidant self-diffusivity in the oxide with a single activation energy. We describe the unified simula-

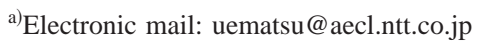

tion of both wet and dry oxidation using a consistent set of parameters.

\section{MODELS}

We have proposed the basic model based on the following concepts: ${ }^{10-12,16} \mathrm{~A}$ large number of $\mathrm{Si}$ atoms $(\sim 1 \%$ of the oxidized $\mathrm{Si}$ atoms) are emitted from the interface and most of them diffuse into the oxide. The emitted $\mathrm{Si}$ atoms in the oxide govern the oxidation rate because the existence of high-concentration $\mathrm{Si}$ atoms should prevent the emission of new $\mathrm{Si}$ atoms at the interface. These concepts lead to the point that the oxidation reaction in the oxide, which absorbs the emitted $\mathrm{Si}$ atoms, controls, or modulates, the oxidation rate at the interface. The oxidation models that consider the $\mathrm{Si}$-atom emission in oxide have been proposed in Refs. 7 and 8 , however, they did not include that the emitted $\mathrm{Si}$ atoms affect the oxidation rate at the interface. In contrast, our model takes into account the concept that the emitted $\mathrm{Si}$ atoms in the oxide govern the oxidation rate.

The Si emission model described above leads to the following set of coupled partial differential equations: ${ }^{12}$

$$
\begin{aligned}
& \frac{\partial C_{\mathrm{Si}}}{\partial t}=\frac{\partial}{\partial x}\left(D_{\mathrm{Si}} \frac{\partial C_{\mathrm{Si}}}{\partial x}\right)-R_{1}-R_{2}, \\
& \frac{\partial C_{\mathrm{O}}}{\partial t}=\frac{\partial}{\partial x}\left(D_{\mathrm{O}} \frac{\partial C_{\mathrm{O}}}{\partial x}\right)-R_{1}-R_{2}-R_{3},
\end{aligned}
$$

where $R_{1}, R_{2}$, and $R_{3}$ are the reaction terms that represent the oxidation at the oxide surface, that in the oxide, and the oxidant transfer from the gas to the oxide surface, respectively, such that

$$
\begin{aligned}
& R_{1}=k^{\prime} C_{\mathrm{O}}^{\mathrm{S}} C_{\mathrm{Si}}^{\mathrm{S}}, \\
& R_{2}=\kappa_{1} C_{\mathrm{O}} C_{\mathrm{Si}}+\kappa_{2}\left(C_{\mathrm{O}}\right)^{2} C_{\mathrm{Si}}, \\
& R_{3}=h\left(C_{\mathrm{O}}^{\mathrm{S}}-C_{\mathrm{O}}^{*}\right) .
\end{aligned}
$$


TABLE I. Parameters used in the simulation of dry oxidation.

\begin{tabular}{lll}
\hline \hline Parameter & \multicolumn{1}{c}{ Value (dry oxidation) } & Reference \\
\hline$C_{\mathrm{Si}}^{0}$ & $3.60 \times 10^{24} \exp \left(-1.07 \mathrm{eV} / k_{B} T\right) \mathrm{cm}^{-3}$ & 18 and 19 \\
$C_{\mathrm{O}}^{*}$ & $5.5 \times 10^{16} \mathrm{~cm}^{-3}$ & 3 \\
$D_{\mathrm{Si}}^{\mathrm{SD}}$ & $1.3 \times 10^{1} \exp \left(-4.5 \mathrm{eV} / k_{B} T\right) \mathrm{cm}^{2} \mathrm{~s}^{-1}$ & 20 \\
$D_{\mathrm{O}}^{\mathrm{SD}}$ & $3.20 \times 10^{-8} \exp \left(-1.64 \mathrm{eV} / k_{B} T\right) \mathrm{cm}^{2} \mathrm{~s}^{-1}$ & 12 \\
$\nu$ & $9.44 \times 10^{4} \exp \left(-1.76 \mathrm{eV} / k_{B} T\right)\left[T \leqslant 1000{ }^{\circ} \mathrm{C}\right]$ & 12 \\
& $2.78 \times 10^{2} \exp \left(-1.12 \mathrm{eV} / k_{B} T\right)\left[T \geqslant 1000^{\circ} \mathrm{C}\right]$ & \\
$\kappa_{1}$ & $1.46 \times 10^{-14} \exp \left(-1.55 \mathrm{eV} / k_{B} T\right) \mathrm{cm}^{3} \mathrm{~s}^{-1}$ & 12 and 16 \\
$\kappa_{2}$ & $1.46 \times 10^{-31} \exp \left(-1.55 \mathrm{eV} / k_{B} T\right) \mathrm{cm}^{6} \mathrm{~s}^{-1}$ & 12 and 16 \\
\hline \hline
\end{tabular}

In these Eqs. $(3.1-3.3), C_{\mathrm{Si}}$ and $C_{\mathrm{O}}$ are the concentration of Si interstitials and the oxidant, $D_{\mathrm{Si}}$ and $D_{\mathrm{O}}$ are the diffusion coefficients of Si interstitials and the oxidant in the oxide, $k^{\prime}$ is the oxidation rate of $\mathrm{Si}$ atoms at the oxide surface, $\kappa_{1}$ and $\kappa_{2}$ are the rates of $\mathrm{Si}$ atoms in the oxide, $h$ is the gas phase mass-transfer coefficient, and $C_{\mathrm{O}}^{*}$ is the solubility of the oxidant in the oxide. $C_{\mathrm{Si}}^{\mathrm{S}}$ and $C_{\mathrm{O}}^{\mathrm{S}}$ are the concentration of $\mathrm{Si}$ atoms and the oxidant at the oxide surface. In order to express the reduction of the oxidation rate with the increase of the interfacial concentration of the $\mathrm{Si}$ atoms, we describe the oxidation reaction rate constant at the oxide/silicon interface, $k$, by the decreasing function of $C_{\mathrm{Si}}^{\mathrm{I}}$ as

$$
k=k_{0}\left(1-C_{\mathrm{Si}}^{\mathrm{I}} / C_{\mathrm{Si}}^{0}\right),
$$

where $C_{\mathrm{Si}}^{\mathrm{I}}$ is the $\mathrm{Si}$ interstitial concentration in the oxide around the interface, $C_{\mathrm{Si}}^{0}$ is the maximum concentration of $\mathrm{Si}$ interstitials in the oxide, and $k_{0}$ is the maximum interfacialreaction-rate constant. The boundary conditions for the interstitials and the oxidant at the interface $(x=0)$ are given by

$$
\left.D_{\mathrm{Si}} \frac{\partial C_{\mathrm{Si}}}{\partial x}\right|_{x=0}=-k \nu C_{\mathrm{O}}^{\mathrm{I}} \text { and }\left.D_{\mathrm{O}} \frac{\partial C_{\mathrm{O}}}{\partial x}\right|_{x=0}=k C_{\mathrm{O}}^{\mathrm{I}} \text {, }
$$

where $\nu$ is the emission rate of $\mathrm{Si}$ atoms from the interface and $C_{\mathrm{O}}^{\mathrm{I}}$ is the concentration of the oxidant at the interface. The oxide growth rate is described as

$$
N_{0} \frac{d X}{d t}=k C_{\mathrm{O}}^{\mathrm{I}}
$$

where $N_{0}$ is the number of $\mathrm{SiO}_{2}$ molecules in a unit volume of the oxide and $X$ is the thickness of the oxide layer. Eqs. (1)-(5) were solved numerically by the partial differential equation solver ZOMBIE, ${ }^{17}$ and the oxide thickness, $X$, at each time step is obtained from Eq. (6).

Using this model, we have simulated the whole range of oxide thickness for dry oxidation in a wide range of oxidation temperatures and oxygen pressures, and have explained the initial oxidation enhancement ${ }^{11,12}$ and the sublinear dependence. ${ }^{16}$ The essential parameters used in the simulation are summarized in Table I. $C_{\mathrm{Si}}^{0}$ was estimated from the product of the interstitial segregation coefficient for the oxide/silicon interface ${ }^{18}$ and the equilibrium self-interstitial concentration in silicon. ${ }^{19} \mathrm{We}$ used the $C_{\mathrm{O}}^{*}$ value from Ref. 3 and the experimentally obtained self-diffusivity $D_{\mathrm{Si}}^{\mathrm{SD}}$ ( $=D_{\mathrm{Si}} C_{\mathrm{Si}}^{0} / N_{0}$ ) from Ref. 20. The oxidant self-diffusivity, $D_{\mathrm{O}}^{\mathrm{SD}}\left(=D_{\mathrm{O}} C_{\mathrm{O}}^{*} / N_{0}\right), \nu, \kappa_{1}$, and $\kappa_{2}$, were deduced from the simulation to fit the experimental data. Note that the values

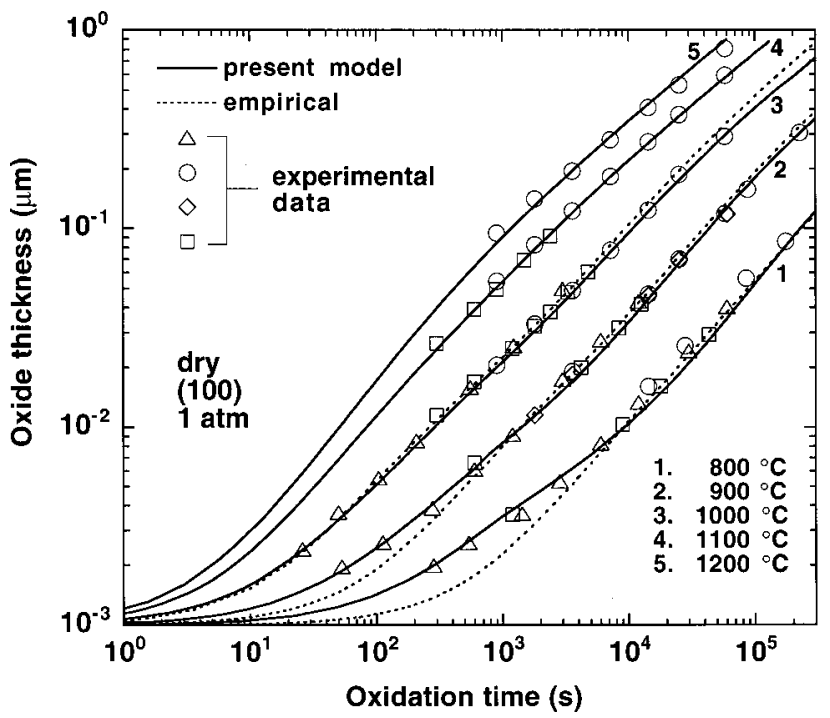

FIG. 1. Simulated (solid lines) and experimental (symbols) oxide thickness for dry oxidation of $\mathrm{Si}(100)$ substrates with the oxygen pressure of 1 atm at $800{ }^{\circ} \mathrm{C}-1200^{\circ} \mathrm{C}$. Experimental data are from Refs. 6 (diamonds), 21 (triangles), 22 (circles), and 23 (squares). Calculated results using the empirical equation and the parameter values given for $T \leqslant 1000^{\circ} \mathrm{C}$ in Ref. 24 are also shown (dashed lines).

of $k_{0}$ for dry oxidation could not be accurately determined because the amount of Si-atom emission in dry oxidation is so high that the oxidation rate is primarily governed by $C_{\mathrm{Si}}^{\mathrm{I}}$ (and thereby $\nu$ ) and the calculated results change only slightly with the variation of $k_{0}$. In addition, the values of $k^{\prime}$ and $h$ are large enough, and the calculated results are quite insensitive to their variations.

The oxide thickness simulated based on our model (solid lines) and that from experiments (symbols) $)^{6,21-23}$ are shown in Fig. 1 for dry oxidation of $\mathrm{Si}(100)$ substrates with the oxygen pressure of 1 atm at $800{ }^{\circ} \mathrm{C}-1200{ }^{\circ} \mathrm{C}$. For comparison, the calculated results using the empirical equation $\left[d X / d t=B /(A+2 X)+C_{2} \exp \left(-X / L_{2}\right)\right]$ and the parameter values $\left(B / A, B, C_{2}\right.$, and $\left.L_{2}\right)$ given for $T \leqslant 1000^{\circ} \mathrm{C}$ in Ref. 24 are included (dashed lines). The empirical model could not fit the thin film regime for $800^{\circ} \mathrm{C}$ and $900^{\circ} \mathrm{C}$, and the second exponential term, $C_{1} \exp \left(-X / L_{1}\right)$, in Ref. 24 is necessary for the fittings. In contrast, we have fit the whole range of the oxide thickness, including the thin film regime, in a wide range of oxidation temperatures. In the thin film regime, the emitted $\mathrm{Si}$ atoms can rapidly leave the interface, and hence the oxidation rate is normal, or not reduced. As the oxide becomes thick, the emitted $\mathrm{Si}$ atoms remain around the interface, and hence the oxidation rate is more likely to be reduced. This is what we have claimed for the initial oxidation enhancement, ${ }^{11}$ the initial oxide growth is normal and the later growth is reduced.

We have also simulated high-pressure oxidation, where the rate shows sublinear dependence on the oxygen pressure. The variation of oxygen pressure, $P$ (atm), is described only by multiplying the values of $C_{\mathrm{O}}^{*}$ and $D_{\mathrm{O}}^{\mathrm{SD}}$ for 1 atm (Table I) by a factor of $P$; other parameters remain unchanged, because oxygen pressure should change only $C_{\mathrm{O}}^{*}$ in proportion to the pressure. Figure 2 shows the simulated oxide thickness for $1-20$ atm at $900{ }^{\circ} \mathrm{C}$ and for 20 atm at $800{ }^{\circ} \mathrm{C}-1000^{\circ} \mathrm{C}$. 


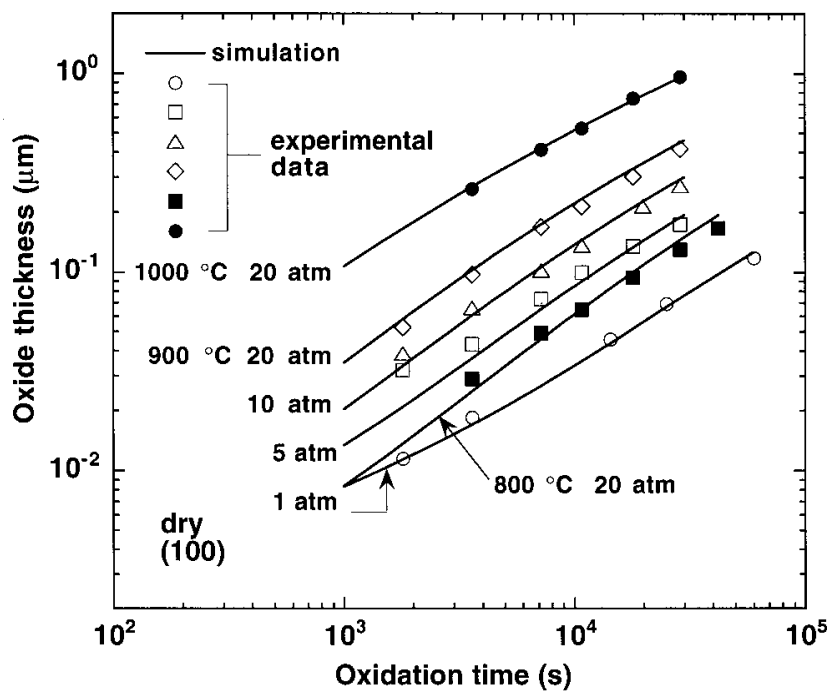

FIG. 2. Simulated (solid lines) and experimental (symbols) oxide thickness for dry oxidation of $\mathrm{Si}(100)$ substrates with the oxygen pressure of $1-20$ atm at $900{ }^{\circ} \mathrm{C}$ and of $20 \mathrm{~atm}$ at $800{ }^{\circ} \mathrm{C}-1000{ }^{\circ} \mathrm{C}$. Experimental data are from Ref. 6.

As oxygen pressure increases, a larger number of $\mathrm{Si}$ atoms is emitted from the interface, which reduces the oxidation rate constant [Eq. (4)]. Therefore, the proportionality of the oxidation rate to oxygen pressure to the power of $n<1$ (Ref. 6) is naturally explained by our model. In contrast, the fitting by the $\mathrm{D}-\mathrm{G}$ theory requires a change of the linear rate constant $(B / A)$ in proportion to oxygen pressure to the power of $n$ with $0.7<n<0.8 .^{6}$

The parameters $D_{\mathrm{O}}^{\mathrm{SD}}$ and $\nu$ deduced from the simulation in Fig. 1 are shown in Fig. 3. For comparison, $B / 2$ corresponding to $D_{\mathrm{O}}^{\mathrm{SD}}$ is also shown, where $B$ is the parabolic rate constant and the values were obtained from the fittings ${ }^{21}$ based on the D-G theory. The $D_{\mathrm{O}}^{\mathrm{SD}}$ in our simulation shows a single activation energy, while that obtained from the D-G theory exhibits a break point in the activation energy at

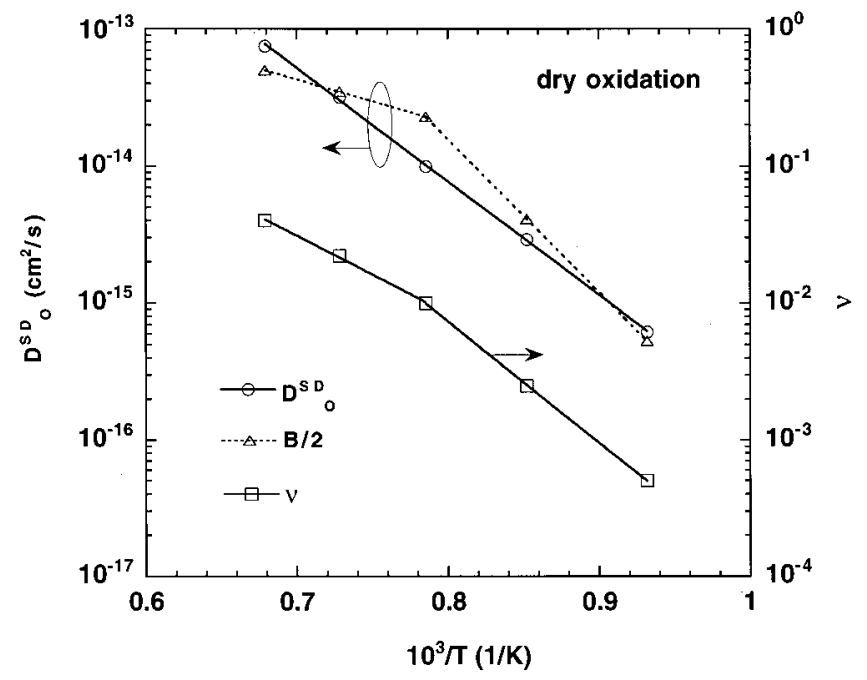

FIG. 3. Arrhenius plot of $D_{\mathrm{O}}^{\mathrm{SD}}$ and $\nu$ deduced from the simulation in Fig. 1. Lines are fittings of the data by the formulas listed in Table I. The values of $B / 2$ ( $B$ from Ref. 21 ), which corresponds to $D_{\mathrm{O}}^{\mathrm{SD}}$, are also shown. around $1000^{\circ} \mathrm{C}$. Moreover, it is the $\mathrm{Si}$ emission rate, $\nu$, that exhibits a break point in its activation energy at around $1000{ }^{\circ} \mathrm{C}$. The break in $\nu$ is attributed to the viscoelastic properties of the oxide because below $960{ }^{\circ} \mathrm{C}$ the oxide interface should be subject to large stress due to a less significant viscous flow ${ }^{25}$ and, in addition $\mathrm{Si}$ atoms are emitted to release the accumulated stress. ${ }^{10}$ Although the break in $B$ was also attributed to the viscoelastic properties of the oxide, ${ }^{21}$ the interfacial Si emission is supposed to be more sensitive to the stress than the transport process of the oxidant, and hence the break in $\nu$ is more likely than that in $B$.

\section{SIMULATION OF WET OXIDATION}

As described above, a unified simulation of dry oxidation has been done based on the interfacial $\mathrm{Si}$ emission model using physically reasonable parameters. In this section, we simulate wet oxidation in a wide range of oxidation conditions in the same way we did dry oxidation.

The $\mathrm{Si}$ atoms emitted to the substrates induce the formation of OSF, and it is demonstrated later that the formation for wet oxidation is slower than that for dry oxidation. Therefore, the emission rate of $\mathrm{Si}$ atoms from the interface, $\nu$, should be smaller for wet oxidation. This is reasonable from the viewpoint of $\mathrm{Si}$-atom emission, which releases the accumulated stress during oxidation; ${ }^{10}$ due to a more significant viscous flow of the oxide grown by wet oxidation, ${ }^{26}$ the oxide interface for wet oxidation should be subject to smaller stress, and therefore a smaller number of $\mathrm{Si}$ atoms would be emitted from the interface in wet oxidation. In addition, the concentration of the emitted $\mathrm{Si}$ atoms in the oxide around the interface, $C_{\mathrm{Si}}^{\mathrm{I}}$, governs the oxidation rate at the interface [Eq. (4)]. Therefore, the difference in $\nu$ accounts for the different features of wet and dry oxidation (initial oxidation enhancement and pressure dependence).

In order to simulate the substrate orientation dependence [(100) or (111)] of the oxidation with other conditions being fixed, the only change made is that the interfacial values, such as $\nu$, are varied according to the orientation. This is quite reasonable because the transport process and the oxidation in the oxide should be independent of the orientation of underlying substrates. Similarly to Ref. 27 for dry oxidation, the $\nu$ values are deduced from this simulation to fit the experimental data for wet oxidation of (100) or (111) substrates. To simulate high-pressure oxidation, the variation of pressure, $P$ (atm), is described only by multiplying the values of $C_{\mathrm{O}}^{*}$ and $D_{\mathrm{O}}^{\mathrm{SD}}$ for 1 atm by a factor of $P$, as has been done for dry oxidation.

The essential parameters used in the simulation are summarized in Table II. As mentioned in Sec. II, the $k_{0}$ values for dry oxidation could not be accurately determined because the amount of Si-atom emission in dry oxidation is so high that the oxidation rate is primarily governed by $C_{\mathrm{Si}}^{\mathrm{I}}$ and the calculated results change only slightly with the variation of $k_{0}$. For wet oxidation, in contrast, the amount of Si-atom emission is smaller and $C_{\mathrm{Si}}^{\mathrm{I}}$ is not large enough to significantly reduce the oxidation rate from $k_{0}$. Therefore, the simulated results critically depend on the $k_{0}$ values, especially at short oxidation times, where $C_{\mathrm{Si}}^{\mathrm{I}}$ is still not large 
TABLE II. Parameters used in the simulation of wet oxidation.

\begin{tabular}{ll}
\hline \hline Parameter & \multicolumn{1}{c}{ Value (wet oxidation) } \\
\hline$C_{\mathrm{Si}}^{0}$ & $1.8 \times 10^{25} \exp \left(-1.07 \mathrm{eV} / k_{B} T\right) \mathrm{cm}^{-3}$ \\
$C_{\mathrm{O}}^{*}$ & $3.4 \times 10^{19} \mathrm{~cm}^{-3}[$ Ref. 3] \\
$D_{\mathrm{Si}}^{\mathrm{SD}}$ & $6.5 \times 10^{1} \mathrm{exp}\left(-4.5 \mathrm{eV} / k_{B} T\right) \mathrm{cm}^{2} \mathrm{~s}^{-1}$ \\
$D_{\mathrm{O}}^{\mathrm{SD}}$ & $5.35 \times 10^{-10} \exp \left(-0.78 \mathrm{eV} / k_{B} T\right) \mathrm{cm}^{2} \mathrm{~s}^{-1}$ \\
$k_{0}$ & $k_{0}(100)=6.38 \times 10^{3} \mathrm{exp}\left(-2.05 \mathrm{eV} / k_{B} T\right) \mathrm{cm} \mathrm{s}^{-1}$ \\
& $k_{0}(111)=1.7 \times k_{0}(100)$ \\
$\nu$ & $\nu($ wet, 100$)=0.2 \times \nu($ dry, 100$)[\nu($ dry, 100$) ;$ see Table I $]$ \\
& $\nu($ wet, 111$)=0.5 \times \nu($ wet, 100$)$ \\
$\kappa_{2}$ & $6.43 \times 10^{-40} \exp \left(-0.71 \mathrm{eV} / k_{B} T\right) \mathrm{cm}^{6} \mathrm{~s}^{-1}$ \\
\hline \hline
\end{tabular}

enough. We mention that, in contrast to $k_{0}, \nu$ at high temperatures for wet oxidation could not be as accurately determined as for dry oxidation due to the small amount of emission; multiplying $\nu$ by a factor of 1.5 leads to the decrease of $X$ by a few percent at $1100^{\circ} \mathrm{C}$ and by less than $1 \%$ at $1200{ }^{\circ} \mathrm{C}$. Concerning the oxidation of $\mathrm{Si}$ atoms in the oxide [Eq. (3.2)], only the $\kappa_{2}$ term is taken into account because two water molecules react with one $\mathrm{Si}$ atom for the oxidation (and hence $R_{2}$ in Eq. (2) is doubled). We used the $C_{\mathrm{O}}^{*}$ value from Ref. 3, and $D_{\mathrm{O}}^{\mathrm{SD}}, \kappa_{2}, k_{0}$, and $\nu$ were deduced from this simulation to fit the experimental data. The maximum concentration of $\mathrm{Si}$ interstitials in the oxide, $C_{\mathrm{Si}}^{0}$, and the Si-atom self-diffusivity, $D_{\mathrm{Si}}^{\mathrm{SD}}$, were also deduced. It should be noted, however, that these values are closely related to $\nu^{11}$ and the deduced $C_{\mathrm{Si}}^{0}$ and $D_{\mathrm{Si}}^{\mathrm{SD}}$ values actually depend on the $\nu$ value used in the simulation [e.g., doubling both $\nu$ and $C_{\mathrm{Si}}^{0}$ (and thereby $D_{\mathrm{Si}}^{\mathrm{SD}}$ ) leads to identical results].

\section{RESULTS AND DISCUSSIONS}

The oxide thickness simulated in this study (dashed and solid lines) and that from experiments (symbols) $)^{2,4,5}$ are shown in Figs. 4-6 for wet oxidation of $\mathrm{Si}(100)$ and (111) substrates with $1 \mathrm{~atm}$ pressure at $800^{\circ} \mathrm{C}-1200^{\circ} \mathrm{C}, 1-20 \mathrm{~atm}$

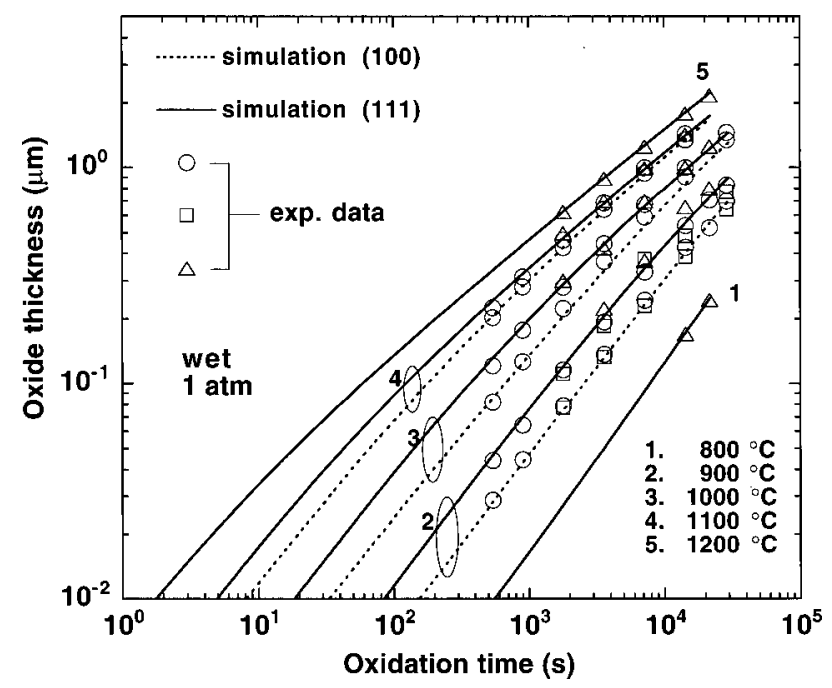

FIG. 4. Simulated (dashed and solid lines) and experimental (symbols) oxide thickness for wet oxidation of $\mathrm{Si}(100)$ and (111) substrates with the pressure of 1 atm at $800{ }^{\circ} \mathrm{C}-1200{ }^{\circ} \mathrm{C}$. Experimental data are from Refs. 2 (triangles), 4 (circles), and 5 (squares).

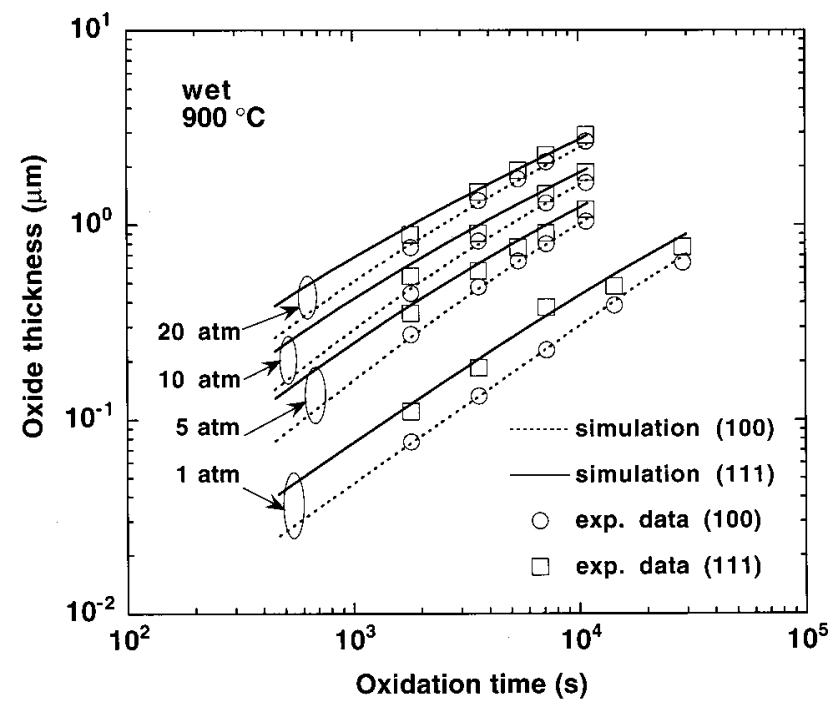

FIG. 5. Simulated (dashed and solid lines) and experimental (symbols) oxide thickness for wet oxidation of $\mathrm{Si}(100)$ and (111) substrates with the pressure of $1-20 \mathrm{~atm}$ at $900{ }^{\circ} \mathrm{C}$. Experimental data are from Ref. 5.

at $900{ }^{\circ} \mathrm{C}$, and $20 \mathrm{~atm}$ at $800{ }^{\circ} \mathrm{C}-1000^{\circ} \mathrm{C}$. Concerning the values of $k_{0}, k_{0}(111)=1.7 \times k_{0}(100)$ was used to fit the data for wet oxidation. This factor of 1.7 is consistent with the ratio between the surface density of the $\mathrm{Si}-\mathrm{Si}$ bonds of (111) and (100) substrates available for the reaction with water molecules. ${ }^{1}$ The activation energy of the oxidation rate of $\mathrm{Si}$ atoms in the oxide $\left(\kappa_{2}\right)$ is $0.71 \mathrm{eV}$ for wet oxidation, which is substantially smaller than the $1.55 \mathrm{eV}$ for dry oxidation (Table I). This is attributable to the more significant viscous flow of the wet-grown oxide and thereby to less accumulated stress induced by volume expansion during oxidation. The values of $C_{\mathrm{Si}}^{0}$ and $D_{\mathrm{Si}}^{\mathrm{SD}}$ used for the simulation of wet oxidation are five times those of dry oxidation. This is also attrib-

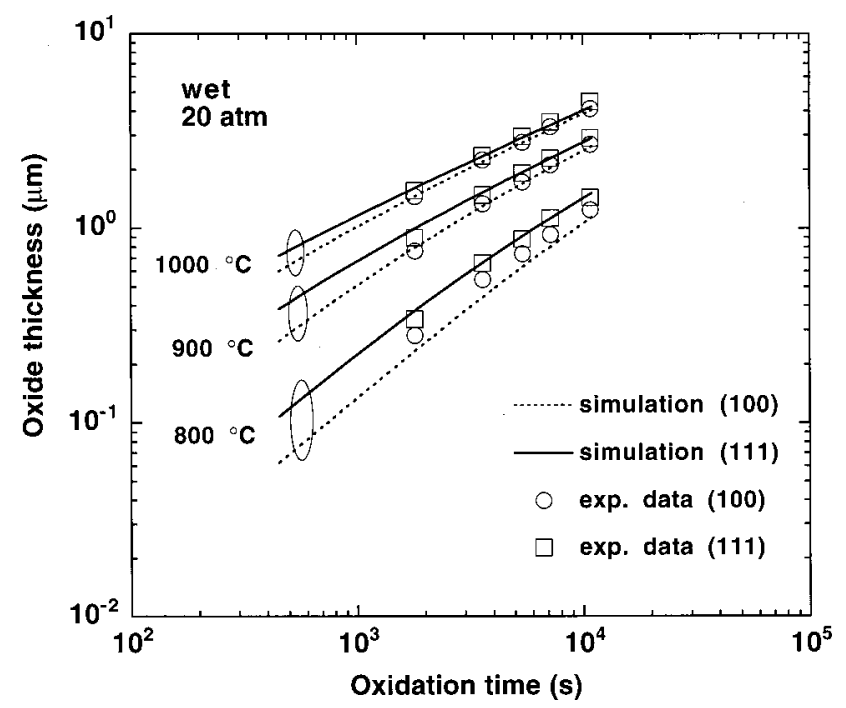

FIG. 6. Simulated (dashed and solid lines) and experimental (symbols) oxide thickness for wet oxidation of $\mathrm{Si}(100)$ and (111) substrates with the pressure of $20 \mathrm{~atm}$ at $800{ }^{\circ} \mathrm{C}-1000{ }^{\circ} \mathrm{C}$. Experimental data are from Ref. 5 . 
uted to a larger flexibility of the wet-grown oxide against the local stress due to the existence of interstitial $\mathrm{Si}$ atoms in the oxide.

Concerning the Si emission rate for (100) substrates, the simulation gives close fits to the experimental oxide thickness using a $\nu$ value for wet oxidation that is less than that for dry oxidation by a factor of five (Table II). This value is not inconsistent with the amount of the Si-atom emission to the substrates estimated from OSF, as follows. In order to compare the $\mathrm{Si}$ emission rate for wet oxidation with that for dry oxidation, the OSF size $(L)$ normalized by the oxide thickness $(X), L / X$, is estimated. The data at only one oxidation time are available for wet oxidation, and the OSF size is about $13 \mu \mathrm{m}$ at $X \sim 0.6 \mu \mathrm{m}$ [for $(100)$ at $1100{ }^{\circ} \mathrm{C}$ and $1 \mathrm{~h}$ ], ${ }^{13}$ leading to $L / X \sim 22$. The time dependence has been reported for dry oxidation ${ }^{13,14}$ and the values of $L / X$ at $1100^{\circ} \mathrm{C}$ are estimated to be $\sim 67$ at $1 \mathrm{~h}, \sim 100$ at $3 \mathrm{~h}$, and $\sim 117$ at 16 h. This variation of $L / X$ with time is attributed to the difference in the time dependence between OSF and oxide growth, ${ }^{14}$ and the $L / X$ value cannot be determined. However, it can be said that $L / X$ for dry oxidation is roughly several times larger than that for wet oxidation, and hence it is not unreasonable that $\nu$ for wet oxidation is about one-fifth that for dry oxidation. Although models to quantitatively explain the time dependence of OSF growth and OED have been proposed, ${ }^{7,8,14}$ further atomic-level studies with firstprinciples calculations are required. For the substrate orientation dependence, we used a $\nu$ value for (111) substrates that is less than that of (100) by a factor of two (Table II). This value is consistent with the amount of the Si-atom emission to the substrates estimated from OSF for wet oxidation; the size of OSF observed in (100) substrates are about twice that in (111). ${ }^{13}$

As mentioned above, we used $\nu($ wet $)=0.2 \times \nu$ (dry). This means that $\nu$ for wet oxidation also exhibits a break point in its activation energy at around $1000^{\circ} \mathrm{C}$ as does $\nu$ for dry oxidation (see Fig. 3). As in dry oxidation, the break point in the activation energy of $\nu$ in wet oxidation is also attributed to the viscoelastic properties of the oxide. ${ }^{25}$ The oxidant self-diffusivity in the oxide, $D_{\mathrm{O}}^{\mathrm{SD}}$, in our simulation shows a single activation energy (Table II). In contrast, the parabolic rate constant $B$, which corresponds to $2 \times D_{\mathrm{O}}^{\mathrm{SD}}$, obtained based on the D-G theory exhibits a break point in the activation energy at around $950{ }^{\circ} \mathrm{C}$. $^{5}$ This break was also attributed to the viscoelastic properties of the oxide. ${ }^{5}$ However, the interfacial Si emission is supposed to be more sensitive to the stress than the transport process of the oxidant, and hence the break in $\nu$ is more likely than that in $B$, as has been discussed for dry oxidation (Sec. II).

\section{COMPARISON WITH DRY OXIDATION}

Figure 7 shows the time dependence of $k$ [the oxidation reaction rate constant at the interface; Eq. (4)] and $X$ deduced from the simulation for wet and dry oxidation of (100) substrates at 1 and $20 \mathrm{~atm}$ and $900{ }^{\circ} \mathrm{C}$. At $t=0, k_{0}=1.0 \times 10^{-5}$ for wet, and $k_{0}=1.4 \times 10^{-3} \mathrm{~cm} \mathrm{~s}^{-1}$ for dry oxidation were used for both 1 and $20 \mathrm{~atm}$. First, we compare the wet and

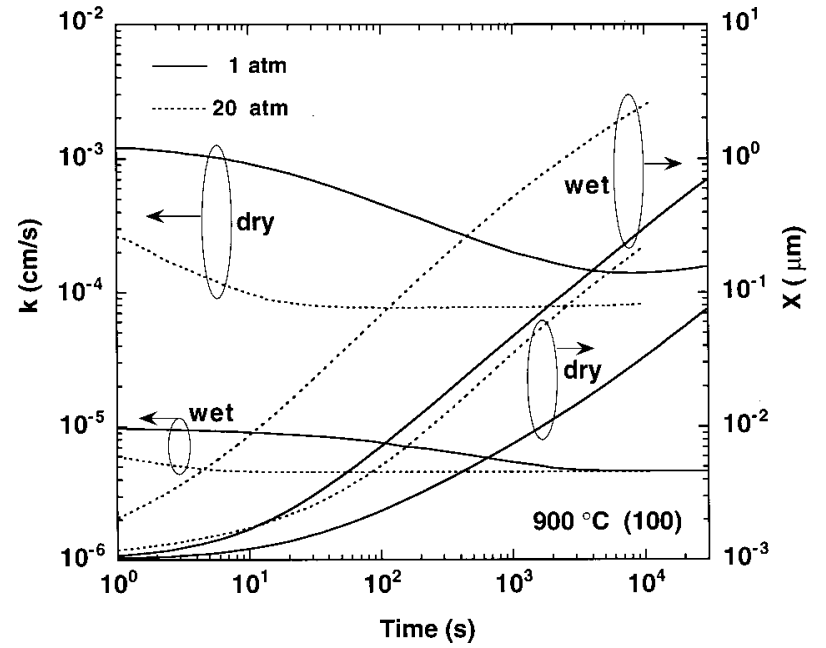

FIG. 7. The time dependence of $k$ and $X$ deduced from the simulation for wet and dry oxidation of (100) substrates with 1 and 20 atm at $900{ }^{\circ} \mathrm{C}$.

dry data for $1 \mathrm{~atm}$ to see the initial oxidation enhancement. The $k$ value for dry oxidation significantly decreases as the oxidation proceeds and $C_{\mathrm{Si}}^{\mathrm{I}}$ increases. This is what we have claimed for the mechanism of the initial oxidation enhancement. ${ }^{11,12}$ For wet oxidation, in contrast, $k$ is not reduced as much as for dry oxidation due to the smaller amount of Si-atom emission. This is the reason the initial oxidation enhancement for wet oxidation is not as significant as for dry oxidation. In addition, Fig. 7 shows that the $k$ values for wet oxidation are reduced only slightly at short oxidation times and, therefore, the calculated results critically depend on the $k_{0}$ values, as just described. We mention that the oxidation rate for wet oxidation is larger even though the $k$ for wet oxidation is smaller because the solubility of water in the oxide is about three orders of magnitude larger than that of oxygen. ${ }^{3}$

Next, we discuss the oxidant pressure dependence of wet and dry oxidation based on Fig. 7. As oxidant pressure increases, a larger number of $\mathrm{Si}$ atoms are emitted from the interface. For dry oxidation, the amount of Si-atom emission is large enough so that $k$ is more likely to be reduced at higher pressures. Therefore, the oxidation rate for dry oxidation is proportional to oxidant pressure to the power of $n$ $<1$ (Ref. 6) as discussed in Sec. II. In contrast, $k$ for wet oxidation is not reduced as much as for dry oxidation and is almost independent of the pressure at oxidation times longer than about $2000 \mathrm{~s}$, which the data for high-pressure oxidation cover. This is the reason the oxidation rate for wet oxidation is proportional to oxidant pressure. ${ }^{5}$

For the initial stage of wet oxidation, the $\mathrm{D}-\mathrm{G}$ theory underestimates the oxide thickness by about $20 \%$ at $<1000$ s for $900{ }^{\circ} \mathrm{C}$. The oxide thickness is more likely to be underestimated for lower temperatures, as pointed out in Ref. 6. In addition, the fitting by the D-G theory requires a double activation energy for $B$, as described above. Moreover, a unified simulation of both wet and dry oxidation cannot be made based on the D-G theory; some modifications are required for the initial oxidation and the pressure dependence 
for dry oxidation. In contrast, our simulation is done using a consistent set of parameters without any empirical modifications for both wet and dry oxidation, and we have fitted the oxide thickness, including the thin film regime, in a wide range of oxidation conditions.

\section{CONCLUSIONS}

A unified simulation of wet oxidation of silicon has been done based on the interfacial $\mathrm{Si}$ emission model. The $\mathrm{Si}$ emission model enables simulation using the oxidant selfdiffusivity in the oxide with a single activation energy, which is more reasonable than a double energy. The differences between wet and dry oxidation, the insignificant initial oxidation enhancement and the linear pressure dependence of the oxidation rate for wet oxidation, are explained by the smaller Si-atom emission rates for wet oxidation. We have simulated the oxide thickness for (100) and (111) substrates in a wide range of oxidation temperatures and pressures. In addition, our simulation based on the Si-atom emission model is done for both wet and dry oxidation using a unified set of parameters.

\section{ACKNOWLEDGMENTS}

The authors thank Professor Yuji Takakuwa, Dr. Masao Nagase, and Dr. Koji Sumitomo for helpful comments. The authors also thank Dr. Yasuo Takahashi and Dr. Katsumi Murase for their useful advice. This work was partly supported by JSPS under Contract No. RFTF96P00203 and by the Telecommunications Advancement Organization of Japan.
${ }^{1}$ J. R. Ligenza, J. Phys. Chem. 65, 2011 (1961).

${ }^{2}$ H. C. Evitts, H. W. Cooper, and S. S. Flaschen, J. Electrochem. Soc. 111, 688 (1964).

${ }^{3}$ B. E. Deal and A. S. Grove, J. Appl. Phys. 36, 3770 (1965).

${ }^{4}$ B. E. Deal, J. Electrochem. Soc. 125, 576 (1978).

${ }^{5}$ R. R. Razouk, L. N. Lie, and B. E. Deal, J. Electrochem. Soc. 128, 2214 (1981)

${ }^{6}$ L. N. Lie, R. R. Razouk, and B. E. Deal, J. Electrochem. Soc. 129, 2828 (1982).

${ }^{7}$ S. T. Dunham and J. D. Plummer, J. Appl. Phys. 59, 2541 (1986).

${ }^{8}$ K. Taniguchi, Y. Shibata, and C. Hamaguchi, J. Appl. Phys. 65, 2723 (1989).

${ }^{9}$ Y. Takakuwa, M. Nihei, and N. Miyamoto, Appl. Surf. Sci. 117, 141 (1997).

${ }^{10}$ H. Kageshima and K. Shiraishi, Phys. Rev. Lett. 81, 5936 (1998).

${ }^{11}$ H. Kageshima, K. Shiraishi, and M. Uematsu, Jpn. J. Appl. Phys., Part 2 38, L971 (1999).

${ }^{12}$ M. Uematsu, H. Kageshima, and K. Shiraishi, Jpn. J. Appl. Phys., Part 2 39, L699 (2000).

${ }^{13}$ S. M. Hu, Appl. Phys. Lett. 27, 165 (1975).

${ }^{14}$ A. M. Lin, R. W. Dutton, D. A. Antoniadis, and W. A. Tiller, J. Electrochem. Soc. 128, 1121 (1981).

${ }^{15}$ P. A. Packan, Ph.D. thesis, Stanford University, 1991.

${ }^{16}$ M. Uematsu, H. Kageshima, and K. Shiraishi, Jpn. J. Appl. Phys., Part 2 39, L952 (2000).

${ }^{17}$ W. Jüngling, P. Pichler, S. Selberherr, E. Guerrero, and H. W. Pötzel, IEEE Trans. Electron Devices 32, 156 (1985).

${ }^{18}$ A. M. Agarwal and S. T. Dunham, J. Appl. Phys. 78, 5313 (1995).

${ }^{19}$ H. Bracht, N. A. Stolwijk, and H. Mehrer, Phys. Rev. B 52, 16542 (1995).

${ }^{20}$ G. K. Celler and L. E. Trimble, Appl. Phys. Lett. 54, 1427 (1989).

${ }^{21}$ H. Z. Massoud, J. D. Plummer, and E. A. Irene, J. Electrochem. Soc. 132, 1745 (1985).

${ }^{22}$ D. W. Hess and B. E. Deal, J. Electrochem. Soc. 124, 735 (1977).

${ }^{23}$ M. Uematsu (unpublished).

${ }^{24}$ H. Z. Massoud, J. D. Plummer, and E. A. Irene, J. Electrochem. Soc. 132, 2685 (1985).

${ }^{25}$ E. P. EerNisse, Appl. Phys. Lett. 30, 290 (1977)

${ }^{26}$ S. M. Hu, J. Appl. Phys. 64, 323 (1988).

${ }^{27}$ M. Uematsu, H. Kageshima, and K. Shiraishi (unpublished). 